\title{
Breve crónica y aplicación de dos criterios de apoyo para la identificación de una clinoptilolita.
}

\section{Brief chronicle and application of two support criteria for the identification of a clinoptilolite.}

\author{
Damián Díaz-Guzmán ${ }^{\mathrm{a}, *}$, Felipe Legorreta-García ${ }^{\text {iDa a }}$, Nelly K. Pérez-González ${ }^{\mathrm{a}}$, María I. Flores-Zamora ${ }^{\mathrm{b}}$ \\ Marissa Vargas-Ramírez iDa, Victor E. Reyes-Cruz iDa \\ a Área Académica de Ciencias de la Tierra y Materiales, Universidad Autónoma del Estado de Hidalgo, 42184, Pachuca, Hidalgo, México. \\ ${ }^{b}$ Facultad de Ingeniería, UACH, Circuito No. 1, Campus Universitario 2, Chihuahua, Chih., 31125
}

\begin{abstract}
Resumen
Los marcos estructurales de la clinoptilolita y heulandita son idénticos al analizarse con la técnica de difracción de rayos X. Esto ha causado confusión desde la mitad del siglo pasado, por lo que, se han hecho grandes esfuerzos para distinguir entre ambos marcos estructurales. Tan grande ha llegado a ser esta confusión que el subcomité en zeolitas de la Asociación Mineralógica Internacional ha manifestado una regla especial para la nomenclatura de estas zeolitas. En este trabajo de investigación se introduce una breve crónica sobre estos esfuerzos, así como, la explicación de los principios básicos que se aplican para identificar si la zeolita es tipo clinoptilolita o heulandita. Se realiza la caracterización de una muestra de zeolita natural por medio de las técnicas de difracción de rayos X (DRX), microscopia electrónica de barrido y análisis elemental semicuantitativo (MEB - EDS), para determinar el tipo de serie de zeolita, usando dos criterios de apoyo: el de estabilidad térmica sugerido por Mumpton, y el parámetro empírico $\Gamma$ sugerido por Valueva, así mismo se determina la relación Si: Al. También se realiza la técnica de fluorescencia de rayos X (FRX) para determinar la composición elemental de elementos principales de la muestra neta. Este trabajo pretende servir como una guía preliminar que aclare las dudas que comúnmente confunden a los iniciados en el estudio de este tipo de zeolitas.
\end{abstract}

Palabras Clave:

Clinoptilolita, difracción de rayos X, Mumpton, Valueva.

\begin{abstract}
The framework of clinoptilolite and heulandite are identical when analyzed with the X-ray diffraction technique. This has caused much confusion since the middle of the last century, so great efforts have been made to distinguish between both frameworks. So great has this confusion become that the zeolite subcommittee of the International Mineralogical Association has stated a special rule for the naming of these zeolites. In this paper we introduce a brief overview of these efforts, as well as an explanation of the basic principles that apply to identifying whether the zeolite is a clinoptilolite or a heulandite. The characterization of a natural zeolite sample is carried out by means of X-ray diffraction (XRD), scanning electron microscopy and semi-quantitative elemental analysis (SEM - SDS), to determine the type of zeolite series, using two support criteria: the thermal stability suggested by Mumpton, and the parameter suggested by Valueva $\Gamma$, the relationship $S i$ : $A l$ is also determined. The X-ray fluorescence technique (XRF) is also used to determine the elemental composition of the main elements of the net sample. This work is intended to serve as a preliminary guide to clarify the doubts that commonly confuse those initiated in the study of this type of zeolites.
\end{abstract}

Keywords:

Clinoptilolite, Diffraction X-ray, Mumpton, Valueva.

\section{Introducción}

La unidad estructural básica de $\mathrm{SiO}_{4}$ está formada de un tetraedro con cuatro iones de oxígeno en las esquinas y en el centro un ion de silicio. Los silicatos se forman uniendo estos tetraedros, compartiendo un átomo de oxígeno entre ellos por medio de un enlace covalente, donde, uno de los electrones de dicho oxígeno es usado por un silicio y el electrón restante por el silicio del otro tetraedro. Existen siete tipos de estructuras de silicatos, entre ellas los tectosilicatos que se expresan de la forma:

\footnotetext{
*Autor para la correspondencia: lestamian@gmail.com marissav@uaeh.edu.mx (Marissa Vargas-Ramírez), reyescruz16@yahoo.com (Víctor Esteban Reyes-Cruz).

Correo electrónico: profe_974@uaeh.edu.mx (Felipe Legorreta-García), genk.pez@gmail.com (Nelly Karina Pérez-González), exitomifz@gmail.com (María I. Flores-Zamora), 
$\left[\mathrm{Al}_{x} \mathrm{Si}_{y} \mathrm{O}_{2(x+y)}\right]^{x-}$, en el que pertenecen el grupo de las zeolitas (Haldar and Tisljar, 2014). Las zeolitas son aluminosilicatos microporosos, su fórmula general simplificada se puede expresar: $M_{x / n}^{n+}\left[\mathrm{Al}_{x} \mathrm{Si}_{1-x} \mathrm{O}_{2}\right] \cdot y \mathrm{H}_{2} \mathrm{O}$. Donde se puede observar que su estructura está constituida por tres partes: el marco estructural de aluminosilicato $\left[\mathrm{Al}_{x} \mathrm{Si}_{1-x} \mathrm{O}_{2}\right]$ que representa los átomos que lo constituyen de manera fija y que están fuertemente unidos, los cationes intercambiables fuera del marco estructural $M_{x / n}^{n+}$ que comúnmente pueden ser $\mathrm{K}^{+}, \mathrm{Na}^{+}, \mathrm{Ca}^{2+} \mathrm{y} \mathrm{Mg}^{2+}$ y, las moléculas de agua $\mathrm{yH}_{2} \mathrm{O}$ (Auerbach et al., 2003) (Gottardi and Galli , 1985).

Las zeolitas se clasifican en especies. Una especie se constituye por tener un marco estructural de tetraedros topológicamente distintivo y una composición característica distintiva, por ejemplo, la laumontita. Las series consisten de dos o más especies que son distinguibles sobre la base del catión más abundante fuera del marco estructural, por ejemplo, la zeolita de especie chabazita dispone de tres series: una en $\mathrm{Ca}$, otra en $\mathrm{Na}$ o en $K$. Cabe resaltar que la relación $S i$ : $A l$ no es una base adecuada para reconocer una especie diferente, ya que, muchas zeolitas tienen una proporción variable, sin embargo, existe una excepción especial y aplica a las zeolitas llamadas heulandita y clinoptilolita. Dicha excepción, se debe al uso arraigado de sus nombres en su historia mineralógica, y también, a la necesidad de reconocer sus características químicas (Coombs et al., 1998).

El objetivo de este trabajo es revisar parte de la historia que motivó al subcomité en zeolitas de la Asociación Mineralógica Internacional (IMA) (Coombs et al., 1998) para formar la regla mencionada anteriormente referida a la distinción de la clinoptilolita y heulandita, que son idénticas por el análisis de la técnica de difracción de rayos X. Así mismo, determinar el tipo de zeolita que corresponde a la muestra denominada $\mathrm{O} 1$ mediante la interpretación de los resultados obtenidos por las técnicas de caracterización de difracción de rayos X (DRX), microscopia electrónica de barrido (MEB), espectrometría de dispersión de energía de rayos $\mathrm{X}$ (EDS) y con ayuda de dos criterios de apoyo: el sugerido por Mumpton y el parámetro empírico $\Gamma$ sugerido por Valueva. Además de determinar la composición elemental de la muestra neta con la técnica de fluorescencia de rayos X (FRX).

\subsection{Evolución cronológica y fundamentos de los criterios de distinción.}

En 1822, H. J. Brooke examinó un mineral encontrado por el coleccionista J. H. Heuland, al cual Brooke nombró heulandita (Brooke, 1822) en consideración a las contribuciones de los especímenes realizadas por dicho coleccionista. Anteriormente este material se consideraba de la misma especie que la estilbita, ya que, de igual manera pertenece a un sistema monoclínico. Sin embargo, Brooke señaló los planos $u$, que Abbé Haüy omitió al realizar sus exámenes cristalográficos anteriormente (figura 1). En 1890, Louis V. Pirsson (Pirsson, 1890) caracterizó un material como mordenita, al cual describe como: isomorfo con heulandita, su descripción fue a causa de tomar en cuenta la similitud química de la mordenita caracterizada por How en 1864, y al hábito cristalino similar a la heulandita caracterizada por Jones Falls donde se observa el plano (010) señalado con la letra $b$ (figura 2).

El 23 de febrero de 1923 se formaba la sociedad mineralógica de Washington, D. C. (Wherry, 1923), constituida por trece mineralogistas inspirados por la organización informal conocida en ese entonces como el Club de Petrolistas. El primer documento presentado en dicha reunión fue por Waldemar. T. Schaller, titulado: Ptilolita and related zeolites. Este evento fue crucial, ya que, fue la primera ocasión que se denominó a la clinoptilolita, y fue al material anteriormente caracterizado por Pirsson, al cual, James D. Dana (Dana, 1854) había aceptado como mordenita cristalizada en su libro A system of mineralogy en 1854. Se denominó como clinoptilolita porque llegaron a la conclusión de que tenía la misma composición que la ptilolita determinada por Cross y Eakins en 1886 y, además, era monoclínica como la mordenita descrita por How en 1864, además de contar con cristales tabulares, y también, con grandes ángulos de extinción. A causa de estas consideraciones, en 1932 Waldemar T. Schaller (Schaller, 1932) introduce oficialmente el nombre de clinoptilolita basándose en sus grandes ángulos de extinción y la composición química de la entonces ptilolita. No obstante, aunque parecía manifestarse una distinción entre la heulandita y la clinoptilolita, debido a la asociación errónea mencionada anteriormente de la clinoptilolita con la mordenita se desconocía hasta entonces que sus marcos estructurales eran idénticos
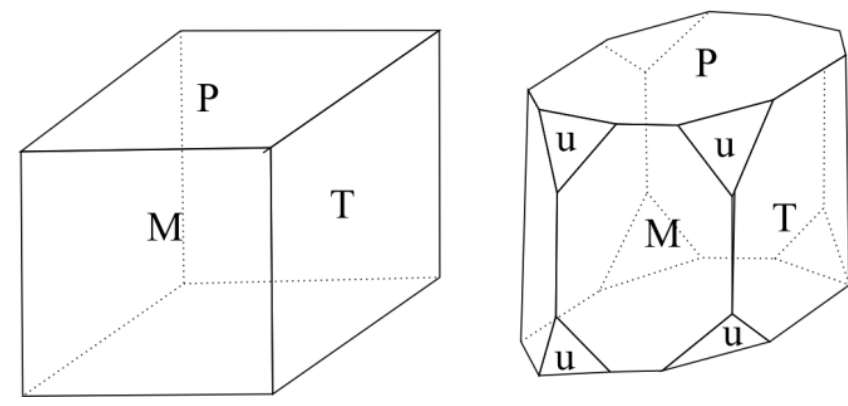

Figura 1: Izquierda, imagen cristalográfica realizada por Abbé Haüy. Derecha, imagen cristalográfica realizada por $\mathbf{H}$. J. Brooke, en la que señaló los planos $u$ que Abbé omitió anteriormente. Por H.J. Brooke en 1822.

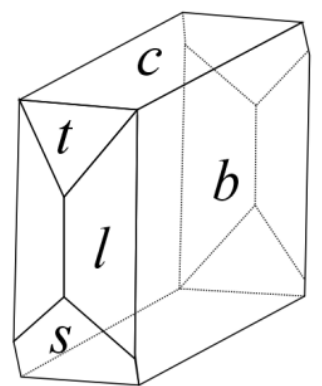

Figura 2: Imagen cristalográfica en el artículo On mordenite de Louis V. Pirsson en 1890, se observa el plano (010) señalado con la letra $b$.

Un año después, M. N. Bramlette y E. Posnjak (Bramlette \& Posnjak, 1933), realizaron los primeros patrones de difracción de rayos X de la clinoptilolita, comparándolos con los de la heulandita, donde mostraron que eran indistinguibles (figura 3). Esta fue la primera prueba de que sus marcos estructurales son idénticos. En 1934 Max H. Hey y F. A. Bannister (Hey and Bannister, 1934), realizaron fotografías de rotación de rayos X, tomadas alrededor del eje $a$ de un cristal de clinoptilolita. Los resultados fueron completamente idénticos a la fotografía de rotación de un cristal de heulandita, que de igual manera fue realizado sobre el eje $a$. Al resultar idénticas y no revelar ninguna diferencia entre ambas los llevó a la conclusión de que son 
miembros de una serie isomorfa, y las diferencias ópticas que existen entre ambas se pueden explicar por la diferencia en la composición, ya que, concluyeron que la clinoptilolita es solo una heulandita rica en sílice. Además, mostraron que su fórmula de la celda unidad para ambas puede ser expresada como $\mathrm{Na}_{x} \mathrm{Ca}_{y} \mathrm{Al}_{x+2 y} \mathrm{Si}_{36-(x+2 y)} \mathrm{O}_{72} \cdot 24 \mathrm{H}_{2} \mathrm{O}$. Ese mismo año W. $\mathrm{H}$. Taylor (Taylor, 1934) propone que la heulandita está formada por un arreglo de tetraedros de silicatos debido a la presencia de un corte perfecto en el plano (010)

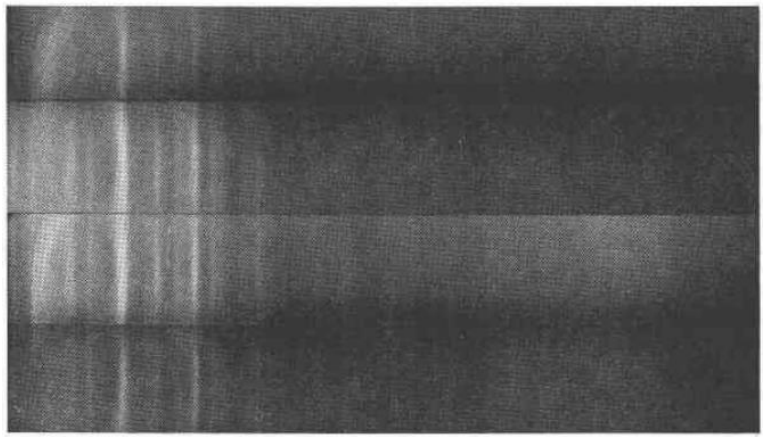

Figura 3: De arriba hacia abajo: Muestra de California, clinoptilolita descrita como mordenita por Pirsson, muestra de Arizona y hasta abajo heulandita. Por Bramlette y Posnjak en 1933.

Desde entonces surgió la confusión al tratar de distinguir a la clinoptilolita de la heulandita. Esto condujo a Frederick A. Mumpton en 1960 (Mumpton, 1960) a realizar un estudio más detallado en el cual concluyó que son minerales estrechamente relacionados, pero distintos en su génesis, su composición, sus propiedades y su estabilidad térmica. Siendo este último el criterio más decisivo para distinguirlas, donde explicó, que si el marco estructural tiene un cambio al ser expuesto a $450{ }^{\circ} \mathrm{C}$ es una heulandita, sin embargo, si a los $700^{\circ} \mathrm{C}$ el marco estructural no sufre ningún cambio, es clinoptilolita. Corroboró estas conclusiones con la ayuda de la difracción de rayos x de las muestras a las temperaturas mencionadas. Además, definió a la clinoptilolita como un mineral de zeolita que tiene una composición cercana a $\left(\mathrm{Na}_{2} \mathrm{O}\right)_{0.70}(\mathrm{CaO})_{0.10}\left(\mathrm{~K}_{2} \mathrm{O}\right)_{0.15}$ $(\mathrm{MgO})_{0.05} \cdot \mathrm{Al}_{2} \mathrm{O}_{3} \cdot 8.5-10.5 \mathrm{SiO}_{2} \cdot 6-7 \mathrm{H}_{2} \mathrm{O}$. Y aceptó la definición declarada en años anteriores, la cual consistía en que la clinoptilolita es una heulandita rica en sílice. Un hecho curioso en la historia es que, en ese mismo número de revista, Brian Mason y L. B. Sand (Mason and Sand, 1960), definieron a la clinoptilolita como una zeolita que pertenece al grupo de la heulandita con la distinción en su composición química que correspondía a $(N a+K)>C a$. En 1966, A. O. Sheppard y H. C. Starkey (Sheppard and Starkey, 1966), demostraron que el intercambio catiónico en ambas zeolitas afecta en su comportamiento térmico, dejando en duda el criterio de Mumpton.

En consecuencia comenzaron los diferentes tipos de pruebas para poder diferenciar entre ambas zeolitas, por mencionar algunas se encuentran la de A.B . Merkle y M. Slaugthter en 1967 (Merkle \& Slaughter, 1968) quienes reexaminaron a la heulandita dando una descripción a detalle del marco tetraédrico describiendo los parámetros de celda con $\mathrm{a}=17.73 \AA \mathrm{A}, \mathrm{b}=17.82 \AA$, $\mathrm{c}=7.43 \AA$, y un sistema monoclínico con $\beta=116^{\circ} 20^{\prime}$. Especificaron la dimensión de sus canales junto con el número de sus miembros de anillos en tres direcciones: paralelo en $a$ los canales están construidos de anillos con 8 miembros provocando dimensiones de $5.40 \times 3.90 \AA$; a $50^{\circ}$ con respecto al eje $a$ se presenta otro canal con anillos de 8 miembros con dimensiones
5.20 x $3.90 \AA ̊$; y paralelo al eje $c$ se forman dos canales, uno con anillos de 10 miembros y el otro con 8 , con dimensiones de 7.05 x $4.25 \AA$ y 4.60 x $3.95 \AA$, respectivamente. Mostraron las similitudes vistas desde el plano $a b$ con el marco de la zeolita tipo mordenita analizada por Meier en $1961 \mathrm{y}$, con el mineral analizado por Perrotta y Smith en 1964 en el plano $b c$, (figura 4). Enfatizaron en el excelente clivaje formado en (010) (figura 5). El reporte de A.B. Merkle y M. Slaugthter aún se toma como referencia en la IZA en conjunto con el realizado en 1972 por A. Alberti (Alberti, 1972), en el cual verificó los grupos espaciales

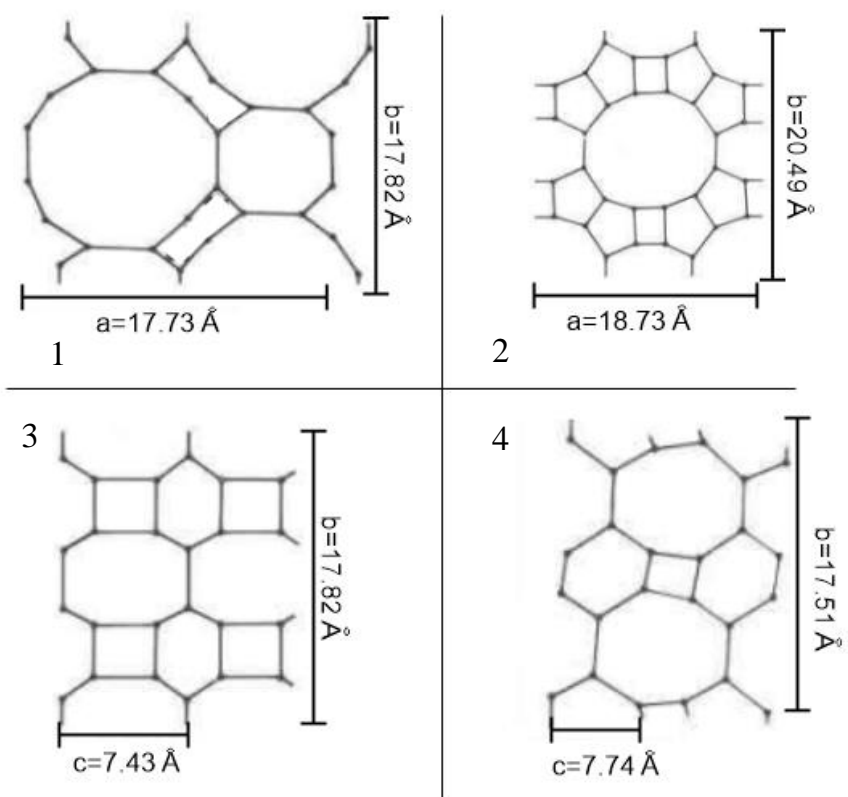

Figura 4. En 1 y 2, los planos $a b$, de la heulandita y mordenita, respectivamente, se puede notar la similitud en el anillo formado por 12 miembros. En 3 y 4, los planos bc de la heulandita y brewsterita, respectivamente, de igual manera se puede notar la similitud entre ambos anillos. Merkle y M. Slaugthter, 1968.

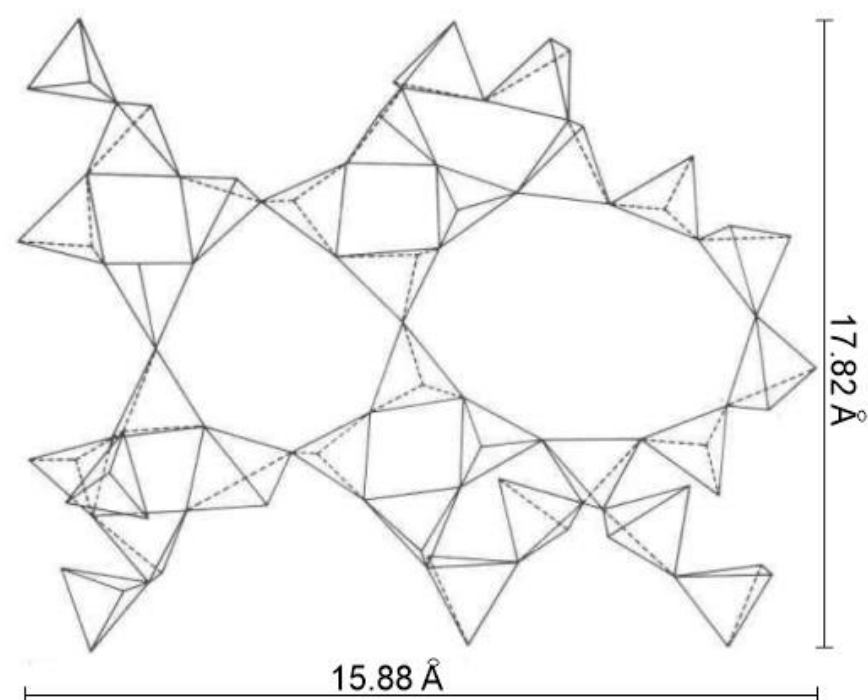

Figura 5. Dibujo esquemático del marco de la heulandita visto desde el plano $b c$, donde se muestra el clivaje (010) y los canales formados por los anillos de 10 y 8 miembros. Merkle y $M$. Slaugthter, 1968. 
En ese mismo año, en 1972, Andrea Alietti (Alietti, 1972) describe las clasificaciones tipo 1, tipo2 y tipo 3 de acuerdo al comportamiento térmico que muestran en un lapso de 12 horas. Describe que el tipo 1 significa la presencia de heulandita, para tipo 2 es una fase intermedia caracterizada como heulandita clinoptilolita, y la fase 3 es clinoptilolita. Así mismo, James R. Boles (Boles, 1972) propone nombrarlas de acuerdo a su relación silicio - aluminio, si $\mathrm{Si} / \mathrm{Al} \geq 4$ es clinoptilolita, si $\mathrm{Si} / \mathrm{Al}<4$ es heulandita. En 1974, Daniel B. Hawkins (Hawkins, 1974) las clasificó en los subgrupos A, B, C, D, E de acuerdo a su incremento en $\mathrm{SiO}_{2}$ y $\mathrm{K}_{2} \mathrm{O}$ o decremento en $\mathrm{Al}_{2} \mathrm{O}_{3}, \mathrm{CaO}$. En 1975, Alberto Alberti (Alberti, 1975) realizó un refinamiento a la estructura de la clinoptilolita concluyendo que la principal diferencia entre ambas zeolitas reside en la carga del marco estructural, reafirmando que la característica más significativa de la clinoptilolita es su alta relación $\mathrm{Si} / \mathrm{Al}$ y su alta resistencia arriba de $700{ }^{\circ} \mathrm{C}$.

En la década de los ochentas se realizaron estudios con respecto al comportamiento térmico pero ahora combinándolo con los efectos que causa la propiedad del intercambio catiónico, en los que se pueden mencionar investigadores como Araya y Dyer (Araya and Dyer, 1981), Alberti y Vezzalini (Alberti and Vezzalini, 1983), David L. Bish (Bish, 1984). Cabe mencionar que en esta década se nota el esplendor sobre el estudio en la zeolita, ya que, surgen libros como el realizado por Gottardi y Galli, en el cual describen desde la génesis, historia, nomenclatura, cristalografía, propiedades químicas, ópticas, físicas, así como usos y aplicaciones de los diferentes tipos de zeolitas (Gottardi and Galli , 1985), y otro no menos importante, como el realizado para la colección "La ciencia desde México" del Fondo de Cultura Económica: La zeolita una piedra que hierve, de Pedro Bosch e Isaac Schifter en 1988, un libro con el fin de llegar no solo a la comunidad científica sino también de servir como una herramienta divulgativa.

En la siguiente década, surgen estudios que buscan correlacionar parámetros, entre ellos, G. P. Valueva (Valueva, 1994) describe un parámetro empírico llamado $\Gamma$, relacionando los datos de los comportamientos térmicos obtenidos por Alietti en 1997 con las composiciones químicas, este parámetro distingue con precisión las clinoptilolita y heulandita. Debido a todos estos factores, en 1998 se reunió el subcomité en zeolitas de la Asociación Mineralógica Internacional (Coombs et al., 1998) (IMA por sus siglas en ingles), contando con grandes exponentes en el área de la zeolita como Douglas S. Coombs (Ahn et al., 1988) (Jillett and Coombs, 1995) (Coombs, 1955), Alberto Alberti (Alberti, 1972) (Alberti, 1975) (Zanardi et al, 2004), Thomas Armbruster (Armbruster, 1993) (Larsen et al, 2005) (Gunter et al, 1994), Gilberto Artioli (Artioli, 1992) (Artioli, 1994), Ermanno Galli (Galli, 1971) (Galli, 1975) (Galli, 1976), E. Passaglia (Passaglia, 1969) (Passaglia, 1970) (Passaglia, 1975), Richard Sheppard (Sheppard and Fitzpatrick, 1989) (Sheppard and Gude, 1969), entre otros; para recomendar reglas sobre la nomenclatura de la zeolita, las cuales fueron aprobadas por la Comisión en Nuevos Minerales y Nombres de Minerales (CNMMC por sus siglas en ingles), donde se enfatiza el caso especial para estas dos zeolitas.

Desde 1996 hasta la actualidad, la base de datos de estructuras de zeolita ha proporcionado información sobre los tipos de estructuras de zeolita que han sido aprobados por la Comisión de Estructura de la Asociación Internacional de Zeolita (IZA - SC por sus siglas en inglés) (Baerlocher, 2017), que muestra la clasificación de las zeolitas de acuerdo a su marco. Para referirse al marco de la heulandita o la clinoptilolita utiliza el código HEU. Los parámetros de celda para estas dos especies de zeolita utilizados por la IZA son: $a=17.5230 \AA, b=17.6460 \AA, c=$ $7.4010 \AA, \alpha=\gamma=90^{\circ}, \beta=116.104^{\circ}$, la cuales cristalizan en un sistema monoclínico. Estos datos son los mismos que los definidos por Meier y Olson en el Atlas of Zeolite Structure Types de 1978 publicado por la Comisión de estructuras del IZA. De acuerdo a la quinta edición de compilación actualmente revisada del 2001 estos datos siguen vigentes (Baerlocher, 2001). Un rasgo distintivo, como se ha ido mencionando, es que presentan un clivaje perfecto (010) por la presencia de los canales abiertos a las alturas de 0 y $1 / 2$ en el eje $b$ (Taylor, 1934) (Merkle and Slaughter, 1967) (Alberti, 1972) (Koyama and Takeuchi, 1977). Concerniente a su composición química ideal de la heulandita se puede expresar: $\mathrm{Ca}_{4} \mathrm{Na}_{8}\left[\mathrm{Al}_{8} \mathrm{Si}_{28} \mathrm{O}_{72}\right] \cdot 24 \mathrm{H}_{2} \mathrm{O}$ (Merkle and Slaughter, 1967) (Merkle and Slaughter, 1968), y así mismo, para la clinoptilolita: $(\mathrm{Na}, \mathrm{K})_{6}\left(\mathrm{Al}_{6} \mathrm{Si}_{30} \mathrm{O}_{72}\right) \cdot 2 \mathrm{H}_{2} \mathrm{O}$ (Gottardi and Galli, 1985).

\section{Metodología.}

La muestra analizada fue recolectada del municipio de Reyes Etla, del estado de Oaxaca, con coordenadas $17^{\circ} 14^{\prime}$ '01.0' ' latitud norte y $96^{\circ} 48^{\prime} 37.8^{\prime}$ ' latitud oeste. Se caracterizó por medio de las técnicas de difracción de rayos X (DRX), microscopia electrónica de barrido (MEB), espectrometría de dispersión de energía de rayos X (EDS) y fluorescencia de rayos X (FRX). Se catalogó como O1. Para la aplicación de todas las técnicas, el primer proceso consistió en triturar la roca a un tamaño en un rango de $1.19 \mathrm{~mm}-0.42 \mathrm{~mm}$ (figura 6). Para esto, se quebró con mazo; acto seguido, se colocó en una trituradora de quijada y después en una quebradora de rodillos. Finalmente se tamizó entre las mallas -\#16 +\#40 para obtener dicho tamaño. El segundo proceso consistió en un lavado con agua desionizada seguido de un secado por 24 horas.

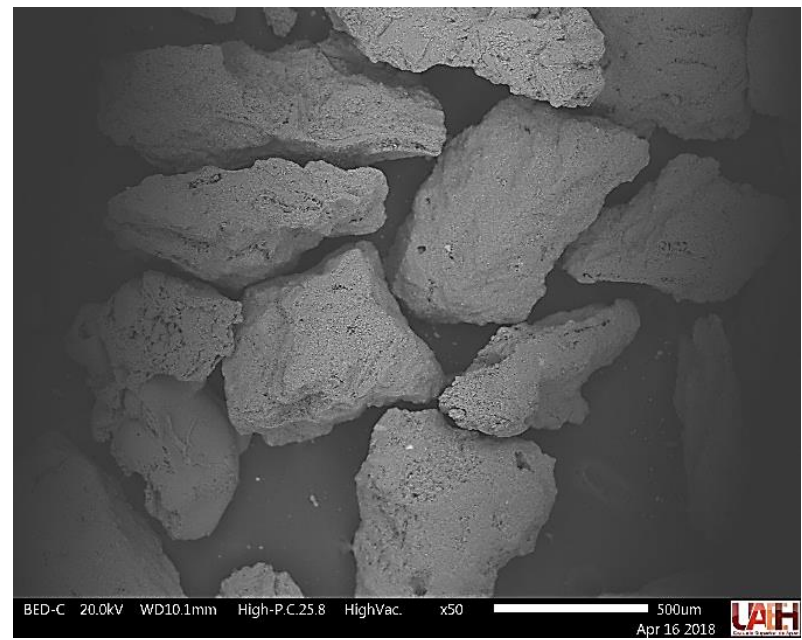

Figura 6: Tamaños de partícula de 01 después de los procesos de trituración, lavado y secado. Se puede apreciar que se encuentran en un rango de $0.42 \mathrm{~mm}$ a $1.19 \mathrm{~mm}$.

\subsection{Difracción de rayos $X(X R D)$.}

Estos análisis se llevaron a cabo con un difractómetro marca INEL modelo Equinox 2000. Con las condiciones de operación: $20 \mathrm{~mA}$ de corriente, un voltaje de $30 \mathrm{kV}$ y una distancia entre los canales de $0.032 \theta$. Con una fuente de radiación de $\operatorname{Co}, \lambda=$ $1.789010 \AA ̊$ y un detector curvo. El tiempo de exposición tanto 
para la muestra principal O1, como para las expuestas a diferentes temperaturas $\left(500^{\circ} \mathrm{C}, 700^{\circ} \mathrm{C}\right.$ y $\left.800^{\circ} \mathrm{C}\right)$ utilizadas para luego determinar el criterio de apoyo de estabilidad térmica propuesto por Mumpton, fue de 20 minutos para cada una, en crisoles de alúmina de alta pureza, para evitar la interacción con la muestra.

La muestra fue pulverizada en un mortero de ágata para después tamizarse en una malla de la serie Tayler \#200, ocupando $2 \mathrm{~g}$ y tomando la muestra que pasó a través de la malla para realizar los análisis cualitativos. Estos análisis fueron realizados con el programa Match versión 3.0 y las fases fueron identificadas usando el PDF - 02 .

Para realizar la distinción estructural por el criterio de apoyo denominado de estabilidad térmica (Mumpton, 1960) (Alietti, 1972) se pulverizó la muestra cómo se mencionó anteriormente. Se tomaron $5 \mathrm{~g}$ para someterse a temperaturas de $500^{\circ} \mathrm{C}, 700^{\circ} \mathrm{C}$ y $800^{\circ} \mathrm{C}$ en una mufla con una rampa de calentamiento de $10^{\circ} \mathrm{C} / \mathrm{min}$, en un tiempo de 4 horas.

\subsection{Microscopia electrónica de barrido $(M E B-E D S)$ y fluorescencia de rayos $X$ (LFRX).}

Para identificar en la morfología el clivaje (010), que es característico en los cristales de heulandita y clinoptilolita (Taylor, 1934) (Merkle and Slaughter, 1967) (IZA, 2020) (Nawaz, 1979), se lleva a cabo un proceso por medio de la técnica de MEB en conjunto con EDS y DRX. Este proceso consiste en explorar las imágenes obtenidas por MEB, para así, seleccionar un cristal y realizarle un análisis cuantitativo elemental por la técnica EDS para determinar la composición química tomando como base la celda unitaria de 72 oxígenos. Posteriormente, con los planos obtenidos por DRX se trazan a través del programa VESTA, para así realizar la comparación del cristal trazado con el cristal seleccionado.

Se utiliza un microscopio electrónico JEOL modelo IT 300. Del cual, se obtiene la micrografía con electrones retrodispersados y con una resolución de x3500 aumentos. Complementariamente, se realizó el análisis elemental de espectrometría de dispersión de energía de rayos X con un detector X-MAX de la casa Oxford Instruments usando un voltaje de $20 \mathrm{keV}$ para determinar la nomenclatura propuesta (Merkle and Slaughter, 1967) (Baerlocher, 2017) (Gottardi and Galli , 1985), así como la aplicación de los criterios de distinción, es decir, la relación $\mathrm{Si}: \mathrm{Al}$ y el parámetro $\Gamma$ (Valueva, 1994).

Para conocer la composición con mayor precisión de la muestra, se utilizaron los datos obtenidos por la técnica de fluorescencia de rayos X (FRX), equipado con un espectrómetro RIGAKU ZSX Primus II. El método de preparación empleado para los elementos mayores fue el de fusión con $50 \% \mathrm{Li}_{2} \mathrm{~B}_{4} \mathrm{O}_{7}-$ $50 \% \mathrm{LiBO}_{2}$, con pérdida por calcinación $(\mathrm{P} \times \mathrm{C})$ a $950^{\circ} \mathrm{C}$.

\section{Análisis y resultados.}

\subsection{Difracción de rayos $X(X R D)$.}

En el patrón de difracción de O1 (figura 7) se identificaron las fases: clinoptilolita, anortita, cuarzo bajo y mordenita. La fase de clinoptilolita fue identificada con la tarjeta PDF 01-083-1261, Koyama 1977 (Koyama and Takeuchi, 1977) y database of zeolite HEU XRD Pattern (Database of zeolite, 2017). Las fases de anortita, cuarzo bajo y mordenita con las tarjetas PDF 01-0710748, PDF $01-079$ - 1906 y PDF $01-078-1766$.

El criterio de apoyo de estabilidad térmica se aplicó con las temperaturas de $25^{\circ} \mathrm{C}, 500^{\circ} \mathrm{C}, 700^{\circ} \mathrm{C}$ y $800^{\circ} \mathrm{C}$ (figura 8). Este criterio se basa en lo propuesto por Mumpton (Mumpton, 1960) que considera las alteraciones del marco estructural con respecto a la temperatura expuesta, en conjunto, con la clasificación propuesta por Alietti (Alietti, 1972). Una alteración significativa del marco después de los $450^{\circ} \mathrm{C}$ se considera de tipo 1 , es decir, heulandita; si la alteración es parcial es de tipo 2, es decir, un intermedio: heulandita-clinoptilolita. Si después de $600^{\circ} \mathrm{C}$ no sufre ninguna alteración es de tipo 3: clinoptilolita. Las intensidades de los planos (010), ( $\overline{2} 01),(001),(\overline{1} 11)$ y $(\overline{4} 22)$ quedaron inalterados después de ser expuestos a $500^{\circ} \mathrm{C}$, mientras los planos (440) y (330) sufrieron una disminución parcial. Al exponerse a $700^{\circ} \mathrm{C}$ los planos que quedaron inalterados fueron: ( 201$),(001),(\overline{4} 22)$ y (111), y los que sufrieron una disminución parcial fueron: (010), (330) y (440). Esto sugiere la presencia de una zeolita tipo 2, es decir, una intermedia. Sin embargo, al exponerse a una temperatura de $800^{\circ} \mathrm{C}$ los picos de la zeolita desparecen, y surgen dos picos más (A) que pertenecen a una fase de hidróxido de calcio con PDF 00 - 044 - 1481, además a esta temperatura los picos de cuarzo bajo (B) aumentaron en intensidad.

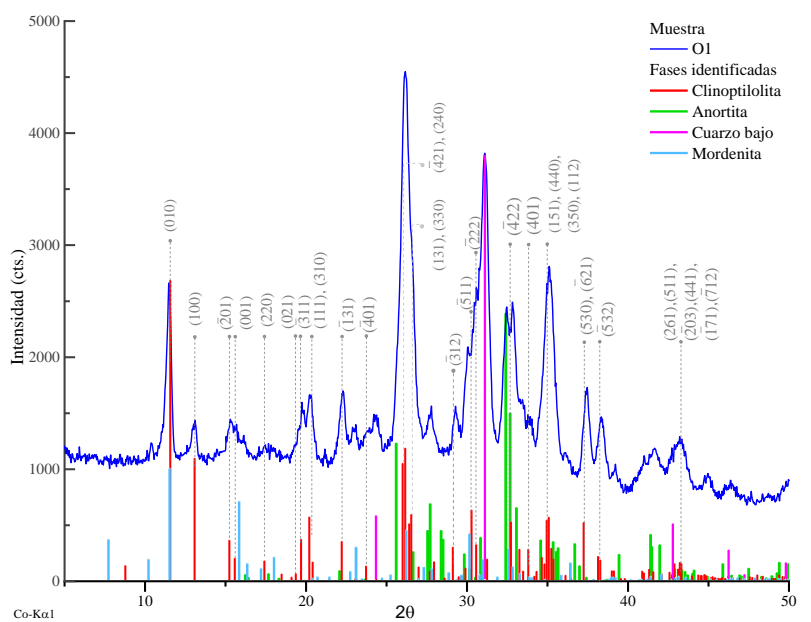

Figura 7. Patrón de difracción de la muestra 01, se han identificado las fases de clinoptilolita (rojo), anortita (verde), cuarzo bajo (rosa) y mordenita (azul). Se señalan los planos cristalográficos identificados pertenecientes a la fase de clinoptilolita con PDF 01-083-1261.

\subsection{Microscopia electrónica de barrido (MEB - EDS) y fluorescencia de rayos $X$ (FRX).}

Los minerales pertenecientes a la familia de la heulandita (ya sean las series de heulandita o clinoptilolita), tienen por lo general

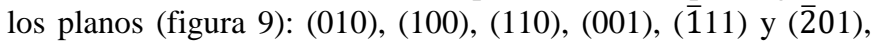
(IZA, 2020) (Nawaz, 1979).

En la micrografía de la figura 10, se observa una partícula cristalina (a) de clinoptilolita que, según la caracterización morfológica (figura 11) presenta los planos (010), (100), (001), $(\overline{2} 01),(\overline{2} 02)$ y $(13 \overline{1})$, típicos del grupo de heulandita. 


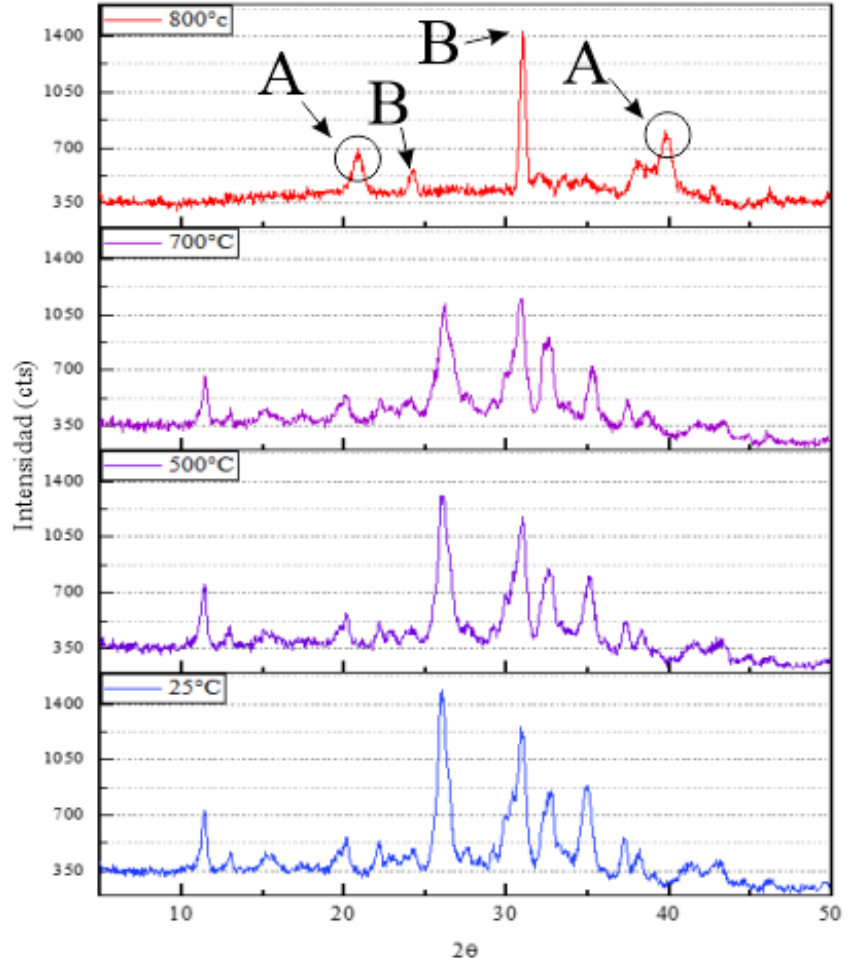

Figura 8. Patrones de difracción a las temperaturas de $25^{\circ} \mathrm{C}$, $5_{00}^{\circ} \mathrm{C}, 7^{\circ 0} 0^{\circ} \mathrm{C}$ y $800^{\circ} \mathrm{C}$ utilizadas para aplicar el criterio de apoyo de estabilidad térmica de Mumpton.

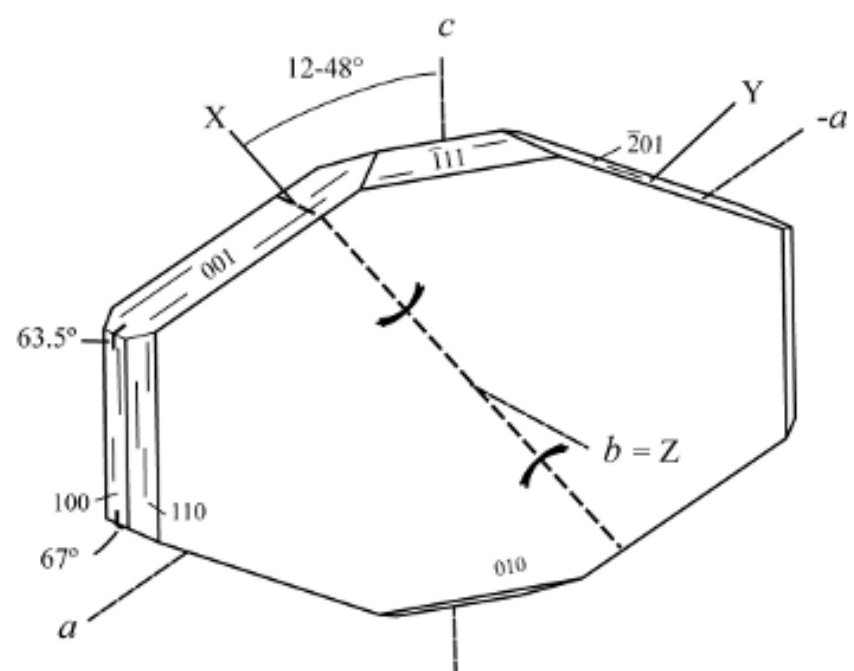

Figura 9: Planos comunes que aparecen en el grupo de la heulandita con su respectiva morfología. Se pueden observar el

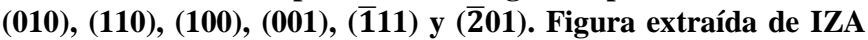
Commission on natural zeolites heulandite (www.izaonline.org/natural/Datasheets/Heulandite/heulandite.htm).

El análisis químico semicuantitativo por espectrometría de dispersión de energía de rayos X (figura 12), arroja la composición media del cristal (a) correspondiente a la figura 10. Se estimó el balance de error (1) obteniéndose un valor de $E \%=5.26$, tal como, lo indican Gottardi y Galli en su libro Natural zeolites para indicar si un análisis químico es aceptable para considerarse como zeolita se debe obtener un valor de balance de error $E<10 \%$ (Gottardi and Galli , 1985). Además, se calculó el parámetro $\Gamma$ (2) propuesto por Valueva (Valueva, 1994) que indicó un valor de $\Gamma=1.13$, el cual, se considera como una zeolita tipo 2, es decir, una intermedia: heulandita - clinoptilolita. Sin embargo, la relación
$\mathrm{Si} / \mathrm{Al}$ arroja un valor de 4.81, el cual, según lo propuesto por la Comisión de Estructura de la Asociación Internacional de Zeolita (IZA - SC) (Baerlocher, 2017) (Coombs et al., 1998) se tiene que es clinoptilolita. La composición estequiométrica obtenida es la siguiente: $\mathrm{Na}_{3.27} \mathrm{~K}_{2}\left(A l_{8.74} \mathrm{Si}_{42.06} \mathrm{O}_{72}\right)$, valorándose como una heulandita rica en sílice, es decir, una clinoptilolita.

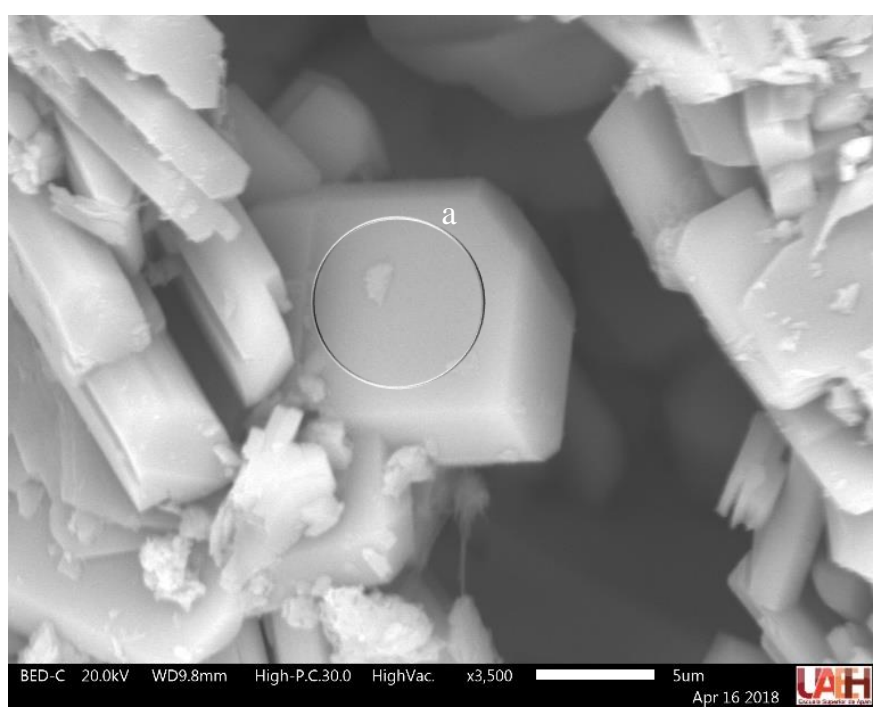

Figura 10: Micrografía de 01, la partícula marcada con (a) es típica del grupo heulandita, partícula que se le realizó su caracterización morfológica, y así mismo, un análisis elemental (área marcada con el circulo). Mide aproximadamente $12 \mu \mathrm{m}$ de largo (plano donde se encuentra el circulo), $9 \mu \mathrm{m}$ de alto sin considerar los perfiles y $3 \mu \mathrm{m}$ de profundidad.

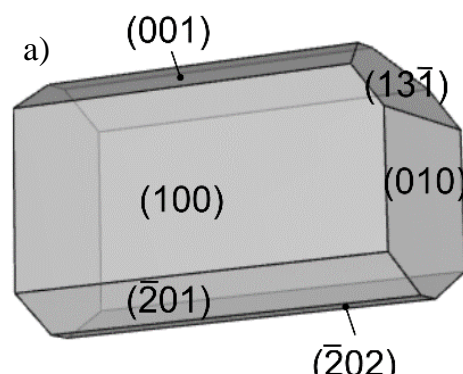

b)

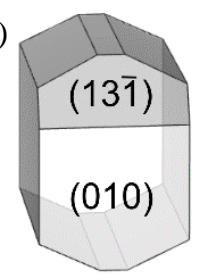

$(\overline{2} 02)$

Figura 11: Caracterización morfológica del cristal observado en los análisis MEB. Se aprecian en (a) los planos típicos del grupo de la heulandita (010), (100), (001), $(\overline{201})$. Y en (b) una vista frontal de los planos (13ī) y (010).

$$
\begin{aligned}
& E \%=\frac{(A l+F e)-(L i+N a+K)-2(M g+C a+S r+B a)}{(L i+N a+K)+2(M g+C a+S r+B a)} \times 100 \\
& \Gamma=C a / A l+0.115 \times A l
\end{aligned}
$$

Al realizarse los análisis elementales mediante FRX se obtiene la composición media (\% masa) expresada como oxido (tabla 1). Se estima que al menos el $80.198 \%$ de la muestra en bruto pertenece a los aluminosilicatos, además un $10.73 \%$ se debe a la humedad propia de los aluminosilicatos hidratados y arcillas. 


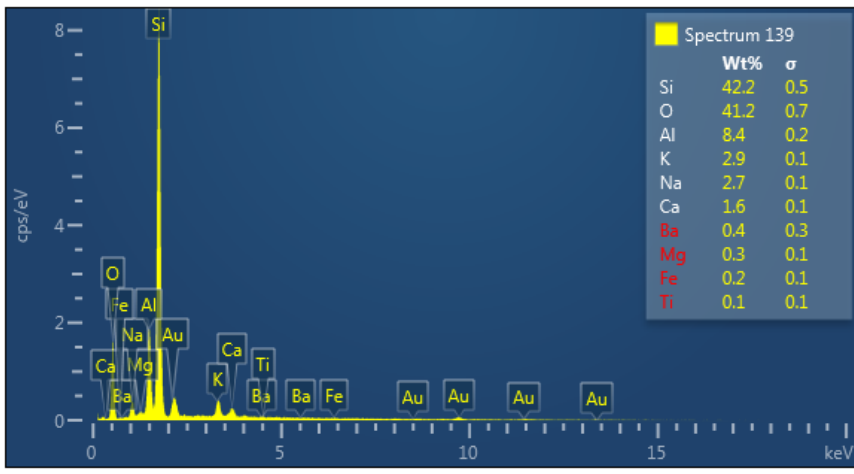

Figura 12: Análisis composicional del cristal seleccionado de la figura 10 con el equipo complementario EDS. Del cual se tomó como base la celda unitaria de 72 oxígenos para determinar el parámetro empírico $\Gamma$ y la relación $\mathrm{Si}: A l$.

Tabla 1: Análisis FRX de la muestra O1.

\begin{tabular}{cc}
\hline Compuesto & \% masa \\
\hline $\mathrm{SiO}_{2}$ & 67.916 \\
$\mathrm{TiO}_{2}$ & 0.127 \\
$\mathrm{Al}_{2} \mathrm{O}_{3}$ & 12.282 \\
$\mathrm{Fe}_{2} \mathrm{O}_{3}$ & 1.677 \\
$\mathrm{MnO}$ & 0.043 \\
$\mathrm{MgO}$ & 0.762 \\
$\mathrm{CaO}$ & 1.236 \\
$\mathrm{Na} 2$ & 2.903 \\
$\mathrm{~K}_{2} \mathrm{O}$ & 2.211 \\
$\mathrm{P}_{2} \mathrm{O}_{5}$ & 0.031 \\
$\mathrm{P}_{x} \mathrm{C}$ & 10.73 \\
$\mathrm{Total}$ & 99.918 \\
\hline
\end{tabular}

\section{Conclusiones.}

De la muestra denominada O1 recolectada del municipio de Reyes Etla, del estado de Oaxaca, con coordenadas $17^{\circ} 14^{\prime}$ '01.0', latitud norte y $96^{\circ} 48^{\prime} 37.8^{\prime \prime}$ ' latitud oeste, se detectó con la técnica de difracción de rayos $\mathrm{X}$ que una de sus fases corresponde a la serie heulandita - clinoptilolita. Así mismo, con la técnica de microscopía electrónica de barrido se localizó un cristal, del cual, con la técnica de espectrometría de dispersión de energía de rayos X se calculó el balance de error (Gottardi and Galli, 1985) determinando que es fiable para considerarse como especie zeolítica, ya que se obtuvo un valor de 5.26\%. Tomando como base 72 Oxígenos ( Ostrooumov et al., 2012) se encontró de acuerdo a las bases del criterio de Mumpton (Mumpton, 1960) y el parámetro empírico $\Gamma$ (Valueva, 1994) que se considera como una zeolita tipo 2, sin embargo, con la regla \# 3 de la Asociación Mineralógica Internacional (Coombs et al, 1998) se determinó una zeolita tipo clinoptilolita, ya que, se obtuvo un valor en su relación $\mathrm{Si} / \mathrm{Al}$ de 4.81 .

\section{Referencias.}

Ahn, J. H., Peacor, D. R., Coombs, D. S. (1988). Formation mechanisms of Illite, chlorite and mixed-layer illite - chlorite in triassic volcanogenic sediments from the Southland Syncline, New Zealand. Contributions to Mineralogy and Petrology, 99(1), 82-89.
Alberti , A. (1972). On the crystal structure of the zeolite heulandite. TMPM Tschermaks Petr. Mitt, 18, 129-146. DOI: 10.1007/BF01081798

Alberti , A. (1975). The crystal structure of two clinoptilolites. TMPM Tschermaks Petr. Mitt, 22, 25-37. DOI:10.1007/BF01081301

Alberti, A., Vezzalini, G. (1983). The thermal behaviour of heulandites: A structural study of the dehydration of Nadap heulandite. TMPM Tschermaks Petr. Mitt, 31, 259-270. DOI:10.1007/BF01081372

Alietti, A. (1972). Polymorphism and crystal-chemistry of heulandites and clinoptilolites. American mineralogist, 57(9-10), 1448-1462.

Araya, A., \& Dyer, A. (1981). Studies on natural clinoptilolites-I: homoionic clinoptilolites. Journal of Inorganic and Nuclear Chemistry, 43, 589-594. DOI:10.1016/0022-1902(81)80508-8

Armbruster, T. (1993). Dehydration mechanism of clinoptilolite and heulandite: single-crystal X-ray study of Na-poor, Ca-, K-, Mg-rich clinoptilolite at $100 \mathrm{~K}$. The American Mineralogist, 78, 260-264.

Artioli, G. (1992). The crystal structure of garronite. American Mineralogist, 77(1-2), 189-196.

Artioli, G. (1994). Gobbinsite from Magheramorne quarry, Northern Ireland. Mineralogical $\quad$ Magazine, 58(393), 615-620 DOI:10.1180/minmag.1994.058.393.10

Auerbach, S. M., Carrado, K. A., Dutta, P. K. (2003). Handbook of zeolite science and technology . Marcel Dekker, Inc.

Baerlocher, C. (2017). Database of Zeolite Structures. Obtenido de http://www.iza-structure.org/databases/

Baerlocher, C., Meier, W., Olson, D. (2001). Atlas of zeolite framework types ( $5^{\circ}$ ed.). (S. C. Association, Ed.) Elseiver.

Bish, D. (1984). Effects of exchangeable cation composition on the thermal expansion/contraction of clinoptilolite. Clays and Clay Minerals, 32(6), $444-452$.

Boles, J. R. (1972). Composition, optical properties, cell dimensions, and thermal stability of some heulandite group zeolites. The American Mineralogist, 57(9-10), 1463-1493.

Bramlette, M. N., Posnjak, E. (1933). Zeolitic alteration of pyroclastics. American Minerologist, 18(4), 167-171.

Brooke , H. J. (1822). On the comptonite of Vesuvius, the brewsterite of Scotland, the stilbite and the heulandite.

Chipera, S. J., Apps, J. A. (2001). Geochemical stability of natural zeolites En Natural zeolites: occurrence, properties, applications (págs. 117 - 161).

Coombs, D. (1955). X-ray observations on wairakite . Mineral. Mag., 30, 699 708 .

Coombs, D. S., Alberti , A., Armbruster , T., Artioli , G., Colella, C., Galli , E., . . . Vezzalini , G. (1998). Recommended nomenclature for zeolite minerals: report of the subcommittee on zeolites of the International Mineralogical Association,Commission on New Minerals and Mineral Names. Mineralogical Magazine, 62(4), 533-571.

Costafreda Mustelier, J. L., Martín Sánchez, D. A., Costafreda Velázquez, J. L. (2018). Las zeolitas naturales de Iberoamérica . Madrid España: Fundación Gómez Pardo.

Dana, J. (1854). A system of mineralogy, comprising the most recent discoveries. New York And London.

Database of zeolite. (2017). Obtenido de http://www.iza-structure.org/IZASC/pow_pat.php?STC=HEU\&ID=HEU_1

Galli, E. (1971). Refinement of the crystal structure of stilbite. Acta $\begin{array}{llll}\text { crystallographica } & \text { section } & \text { B }, & \text { 27(4), }\end{array}$ DOI: $10.1107 /$ S056774087100298X

Galli, E. (1975). Crystal structure refinement of mazzite. Rend. Soc. It. Mineral. Petrol., 31, 599-612.

Galli, E. (1976). Crystal structure refinement of edingtonite. Acta crystallographica section B, 1976. DOI:10.1107/S0567740876006109

Gottardi, G., \& Galli , E. (1985). Natural zeolites . Verlag Berlin Heidelberg: Springer.

Gunter, M. E., Armbruster , T., Kohler , T., \& Knowles , C. R. (1994). Crystal structure and optical propertiesof $\mathrm{Na}$ - and $\mathrm{Pb}$-exchanged heulandite-group zeolites. American Mineralogist, 79(7-8), 675-682.

Haldar, S., \& Tisljar, J. (2014). Introduction to mineralogy and petrology. Elseiver.

Hawkins, D. (1974). Statistical analyses of the zeolites clinoptilolite and heulandite. Contributions to mineralogy and petrology, 45(1), 27-36. DOI:10.1007/BF00371135

Hey, M. H., Bannister, F. A. (1934). Studies on the zeolites. Part VII . Clinoptilolite, a silica-rich variety of heulandite. Mineralogical magazine and journal of the mineralogical society,, 23(145), 556-559. DOI:10.1180/minmag.1934.023.145.03

IZA. (2017). IZA Commission on natural zeolites heulandite. Obtenido de http://www.iza-online.org/natural/Datasheets/Heulandite/heulandite.htm 
Jillett, D., Coombs, D. (1995). Clinoptilolite in the ashley mudstone, Waihao river section, South Canterbury, New Zealand. New Zealand Journal of Geology and Geophysics, 38(2), 263-265.

Koyama , K., Takeuchi, Y. (1977). Clinoptilolite: the distribution of potassium atoms and its role in thermal stability. Zeitschrift für Kristallographic , $145,216-239$.

Larsen, A. O., Nordrum, F. S., Döbelin, N., Armbruster, T., Petersen, O. V., Erambert, M. (Enero de 2005). Heulandite-Ba, a new zeolite species from Norway. Eur. J. Mineral, 17, 143-153. DOI: 10.1127/09351221/2005/0017-0143

Mason, B., Sand, L. B. (1960). Clinoptilolite from Patagonia the relationship between clinoptilolite and heulandite. American minerologist, 45(3-4), 341-350.

Merkle, A. B., Slaughter, M. (1967). The crystal structure of heulandite (Ca, $\mathrm{Na} 2)[\mathrm{Al} 2 \mathrm{Si} 7 \mathrm{O} 18] \cdot 6 \mathrm{H} 2 \mathrm{O}$. American Mineralogist, 52(1-2), 273-276.

Merkle, A. B., Slaughter, M. (1968). Determination and refinament of the structure of heulandite. American mineralogist, 53(7-8), 1120-1138.

Mumpton, F. A. (1960). Clinoptilolite redefined. American Mineralogist, 45(3-4), 351-369.

Nawaz, R. (1979). Crystallography and optical properties of heulandite. Journal of earth sciences, 2(2), 185-187.

Ostrooumov, M., Cappelletti, P., \& de'Gennaro, R. (2012). Mineralogical study of zeolite from New Mexican deposits (Cuitzeo area, Michoacan, Mexico). Applied clay science, 55, 27-35.
Passaglia, E. (1969). Roggianite, a new silicate mineral. Clay Minerals, 8(1), 107-111.

Passaglia, E. (1970). The crystal chemistry of chabazites. American mineralogist, 55(7-8), 1278-1301.

Passaglia, E. (1975). The crystal chemistry of mordenites. Contr. Mineral. and Petrol., 50, 65-77. DOI: 10.1007/BF00385222

Pirsson, L. V. (1890). On mordenite. 232-237.

Schaller, W. T. (1932). The mordenite-ptilolite group; clinoptilolite, a new species. American Mineralogist, 17(4), 128-134.

Sheppard, A. O., Starkey, H. C. (1966). The effects of exchanged cations on the thermal behavior of heulandite. Mineral Soc India, 155-158.

Sheppard, R., Fitzpatrick, J. (1989). Phillipsite from silicic tuffs in saline, alkaline-lake deposits. Clays Clay Minerals, 37, 243-247.

Sheppard, R., Gude, A. (1969). Chemical composition and physical properties of the related zeolites offretite and erionite. Am. Mineral., 54, 875-886.

Taylor, W. H. (1934). The nature and properties of aluminosilicate framework structures. Royal Society, 145, 80-103. DOI: 10.1098/rspa.1934.0083

Valueva, G. P. (1994). Chemical parameters for classification of heulandites. Russian Geol Geophys, 35, 1-4.

Wherry, E. T. (1923). The mineralogical society of Washington, D.C. American Mineralogist, 8(5), 93-95.

Zanardi, S., Alberti , A., Cruciani , G. (2004). Crystal structure determination of zeolite $\mathrm{Nu}-6(2)$ and its layered precursor $\mathrm{Nu}-6(1)$. Angewandte chemie international edition, 43(37), 4933-4937. 